\title{
Multiple Choice Questions
}

1. Genetic mutation which is NOT characteristically associated with Dravet syndrome is
A) $\mathrm{SCN} 2 \mathrm{~A}$
B) $\mathrm{SCN} 1 \mathrm{~B}$
C) CACNA $1 \mathrm{H}$
D) GABARG2

2. All are true facts about Ring 20 chromosome syndrome except
A) NCSE of frontotemporal origin
B) Microcephaly is usual accompaniment
C) Nocturnal CSWS pattern is not a point of exclusion
D) Profound intellectual dysfunction can occur

3. Absence seizures are seen in what percentage of JME
A) $5 \%$
B) $10 \%$
C) $30 \%$
D) $50 \%$

4. Mechanism of action of Perampanel is through
A) Potassium channel blocking
B) Prolonging sodium channel inactivation
C) AMPA receptor facilitation
D) AMPA receptor blocking

5. Which among the following differentiates nocturnal frontal lobe epilepsy from parasomnias?
A) Coherent speech with no recall
B) Five or more events in a single night
C) Duration of more than 10 minutes
D) Wandering outside room

6. All are true about autosomal dominant temporal lobe epilepsy with auditory features except
A) Classically associated with lateral temporal atrophy
B) LGI1 gene mutation is the commonest
C) Commonly simple unformed auditory aura
D) Starts in adolescence or early adulthood

7. Which of the following conditions typically has eye closure sensitivity?
A) Dravet syndrome
B) Doose syndrome
C) Benign Rolandic epilepsy
D) Juvenile absence epilepsy

8. Correct statement about high frequency oscillations (HFO) is
A) They can be recorded by normal surface electrodes
B) They refer to excitatory field potentials in normal hippocampus
C) They have high specificity for seizure onset zone
D) They have no relevance to epilepsy surgery

9. Rufinamide has been approved for clinical use in
A) Ohtahara syndrome
B) West syndrome
C) Migrating partial epilepsy of infancy
D) Lennox-Gastaut syndrome

10. The most commonly encountered benign epileptiform variant is
A) Benign sporadic sleep spikes
B) Phantom spikes
C) Rhythmic midtemporal theta bursts of drowsiness
D) Wicket waves

DOI https://doi.org/ 10.1055/s-0038-1669493. ISSN 2213-6320.
Copyright @2018 Indian Epilepsy Society
License terms

(요 (1) $\odot$ 


\section{Answers:}

\section{Answer C: CACNA $1 \mathrm{H}$}

Explanation: Dravet syndrome or severe myoclonic epilepsy of infancy is an epileptic encephalopathy with polymorphic seizure types including focal or generalized seizures, myoclonic seizures, and atypical absences. The most common genetic mutation has been in SCN1A gene but has also been identified with SCN2A, SCN 1B, GABAR G2, and PCDH19 genes. Mutation in CACNA $1 H$ has been associated with childhood absence epilepsy.

Reference: Helbig I, Scheffer IE, Mulley JC, Berkovic SF. Navigating the channels and beyond: unravelling the genetics of the epilepsies. Lancet Neurol 2008;7(3): 231-245

2. Answer B: Microcephaly is the usual accompaniment Explanation: Ring 20 chromosome syndrome manifests as refractory complex partial seizures (CPS), nocturnal frontal lobe seizures, and non-convulsive status epilepticus (NCSE). Unlike other chromosomal syndromes, this entity lacks a specific phenotypic expression; dysmorphic signs are exceptional. Distinct electro-clinical features suggest a frontotemporal origin of the seizures. The nocturnal EEG pattern in $\mathrm{r}(20)$ may also have overlapping features of continuous spike and wave discharges during slow wave sleep (CSWS). Rare cases of r(20) syndrome with dysmorphic features consisting of microcephaly, plagiocephaly, synophrys, genital hypoplasia, dental malocclusions, micrognathia, cauliflower-shaped ears, and coarse facial features with slanting eyelids have been reported. Individuals may have normal cognition despite periods of poorly controlled epilepsy and others may have profound learning disabilities and require help with all aspects of daily life.

Reference: Radhakrishnan A, Menon RN, Hariharan S, Radhakrishnan K. The evolving electroclinical syndrome of "epilepsy with ring chromosome 20." Seizure 2012;21(2):92-97

3. Answer C: $30 \%$

Explanation: JME is perhaps the most easily recognized of the primary generalized epilepsy syndromes with myoclonic jerks and generalized tonic- clonic seizures being the characteristic features with morning preponderance. However, absence seizures are a well-recognized part of JME and when present always predates the other seizure types.

Reference: Panayiotopoulos CP, Obeid T, Tahan AR. Juvenile myoclonic epilepsy: a 5-year prospective study. Epilepsia 1994;35(2):285-296

\section{Answer $\mathbf{D}$ : AMPA receptor blocking}

Explanation: Glutamate is the major excitatory neurotransmitter and plays an important role in the pathogenesis of epilepsy. It acts through AMPA and NMDA postsynaptic receptors in the brain. Perampanel acts a selective non-competitive AMPA receptor antagonist and prevents AMPA-induced increases in intracellular $\mathrm{Ca} 2+$ thus reducing neuronal excitation.

Reference: Shih JJ, Tatum WO, Rudzinski LA. New drug classes for the treatment of partial onset epilepsy: focus on perampanel. Ther Clin Risk Manag 2013;9:285-293

5. Answer B: Five or more events in a single night

Explanation: Abnormal paroxysmal events in sleep may be parasomnias or epileptic seizures. Frontal lobe epilepsy and parasomnias (FLEP) scale helps to differentiate the two entities based on clinical features. In short, younger age of onset, short duration, clustering in a single night, occurrence in the early part of sleep, stereotyped actions, and preserved recall point to nocturnal frontal lobe epilepsy.

Reference: Derry CP, Davey M, Johns M, et al. Distinguishing sleep disorders from seizures: diagnosing bumps in the night. Arch Neurol 2006;63(5):705-709

6. Answer A: Classically associated with lateral temporal atrophy

Explanation: AD temporal lobe epilepsy with auditory feature is a rare hereditary epilepsy syndrome that usually begins in adolescence or early adulthood. In $50 \%$ of families, mutations have been detected in leucine-rich glioma inactivated gene (LGI 1). The most common auditory symptoms are simple and unformed, such as humming, buzzing, or ringing. Seizures have been known to be precipitated by specific sounds. The diagnosis requires exclusion of any structural lesion as well as positive family history.

Reference: Blair RD. Temporal lobe epilepsy semiology. Epilepsy Res Treat 2012;2012:751510. doi: 10.1155/ 2012/751510

7. Answer $\mathbf{D}$ : Juvenile absence epilepsy

Explanation: Eye closure sensitivity (ECS) is a unique EEG phenomenon that is described as the non-incidental appearance of transient epileptic abnormalities following the closure of the eyes for 1 to 4 seconds during a routine EEG recording. This can be observed both in idiopathic focal and generalized epilepsies. Generalized forms of ECS have also been recorded in most of the genetic/ idiopathic generalized epilepsy (IGE) syndromes, such as childhood absence epilepsy (CAE), juvenile absence epilepsy (JAE), and juvenile myoclonic epilepsy (JME), in addition to the proposed syndrome known as eyelid myoclonia with absences (EMA), in which ECS plays a diagnostic role.

Reference: Striano S, Capovilla G, Sofia V, et al. Eyelid myoclonia with absences (Jeavons syndrome): a welldefined idiopathic generalized epilepsy syndrome or a spectrum of photosensitive conditions? Epilepsia 2009;50 Suppl 5:15-19. doi: 10.1111/j.1528-1167.2009.02114.x

8. Answer C: They have high specificity for seizure onset zone Explanation: High-frequency oscillations or ripples are electrical potentials in 80 to $100 \mathrm{~Hz}$ range recorded from normal hippocampus and parahippocampus 
with intracranial macroelectrodes. They reflect normal inhibitory field potentials needed for neuronal synchronization. HFOs in the range of 250 to $500 \mathrm{~Hz}$ are recorded from pathological hippocampus in mTLE. They have high specificity for seizure onset zone and total resection of HFO containing region has good surgical outcome.

Reference: Raghavendra S, Nooraine J, Mirsattari SM. Role of electroencephalography in presurgical evaluation of temporal lobe epilepsy. Epilepsy Res Treat 2012;2012: 204693. doi: $10.1155 / 2012 / 204693$

9. Answer D: Lennox-Gastaut syndrome

Explanation: Rufinamide is a triazole derivative which acts by prolongation of sodium channel inactivation. Rufinamide is especially effective for tonic-atonic seizures in Lennox- Gastaut syndrome, but is subsequently

Dr. Deepak Menon

MD, DM Neurology, DNB Neurology

MNAMS, MRCP(UK)

Fellowship in Epilepsy

Fellowship in Neuromuscular disorder

Certified specialist in Sleep Medicine

Epileptologist, Consultant Neurologist

Kerala Institute of Medical Sciences,

Trivandrum, India proving to be safe and effective in clinical practice for a broad patient population with refractory epilepsy.

Reference: Hsieh DT, Thiele EA. Efficacy and safety of rufinamide in pediatric epilepsy. Ther Adv Neurol Disord 2013;6(3):189-198

10. Answer A: Benign sporadic sleep spikes

Explanation: They fall into six main types: benign sporadic sleep spikes (BSSS), wicket waves, $14-$ and $6-\mathrm{Hz}$ positive spikes, $6-\mathrm{Hz}$ spike and waves, rhythmic temporal theta burst of drowsiness (RTTD), and subclinical rhythmic electrographic discharge of adults (SREDA). They commonly occur during drowsiness and light sleep. The most commonly encountered BEV is BSSS.

Reference: Santoshkumar B, Chong JJ, Blume WT, et al. Prevalence of benign epileptiform variants. Clin Neurophysiol 2009;120(5):856-861

Dr. Sanjeev V. Thomas

Professor of Neurology and Head

R Madhavan Nayar Center for Comprehensive Epilepsy Care

Department of Neurology

Sree Chitra Tirunal Institute for Medical Sciences

and Technology

Trivandrum, India 
